\title{
Some Issues of Methods, Theories, and Experimental Designs ${ }^{1}$ \\ By James C. Cox
}

\section{Introduction}

Smith (2008, p. 23) reports an example that illustrates how easily procedures can introduce "a future" into an experiment. The example is provided by the central result reported in Cox (2000). That paper explores a behavioral phenomenon that has not been included in theories of reciprocity or models of unconditional social preferences: the effects of social context on reciprocity, trust, and altruism. The weak social context treatment includes the investment game and two dictator control games in a triadic design (Cox, 2004) with double blind payoff protocol. The strong social context treatment includes the same three games and double blind protocol and two added features: (a) an unexplained "second task"; and (b) a coin flip random selection of the second task or the first task (investment game or one of the two dictator games) for money payoff. $^{2}$ Introduction of the second task had no significant effect on first movers' behavior in the investment game. But it significantly shifted behavior of second movers in the investment game and dictators in both the first-mover dictator-control game and the second-mover dictator-control game towards more generous giving: information about the existence of the second task caused subjects in these roles to behave as though they were more altruistic. The result is striking because the double blind protocol makes it impossible for a subject to have a reputation with other subjects or the experimenter and, hence, the random decision selection procedure "should" make behavior in the first task independent of information about the existence of the second task.

This example involves two of the themes explored in Smith's (2008) paper, “independence of history and future” and “context irrelevance," that can arise when subjects' multiple decisions within an experiment are not independent. In the following pages, I will

\footnotetext{
${ }^{1}$ Vjollca Sadiraj provided helpful comments on an earlier draft. The National Science Foundation provided financial support (grant number IIS-0630805).

${ }^{2}$ After completing the first decision task, the subjects received instructions for the second task, which was an investment game with three-person groups as decision making agents.
} 
explore related questions that arise in theory and experiments when subjects make multiple decisions. These are questions of experimental design, theoretical interpretation of data, and behavioral impacts that arise from possible nonseparability of utilities: (a) among decision tasks in an experiment; (b) among risky choice options in an experiment; and (c) between decision tasks within an experiment and decision tasks outside the experiment. From a theoretical perspective, the issues appear to be somewhat different when the experiment involves decisions on a lottery space than when it involves decisions on a commodity space. ${ }^{3}$

\section{Independence, Dual Independence, Wealth Effects, and Portfolio Effects}

Many experiments on choice under risk (in a "lottery space”) involve multiple decisions and random selection of one decision for money payoff. The rationale for this method can be illustrated by experiments on the preference reversal phenomenon in which the central question is whether subjects reveal inconsistent preferences in choice tasks and valuation tasks. The literature on the preference reversal phenomenon contains much discussion of methodological questions, which makes it a good example for present purposes.

\subsection{An Illustrative Example: Random Decision Selection in Preference Reversal Experiments}

Consider two simple (usually binary) lotteries $A$ and $B$. Ask the subjects to choose between $A$ and $B$ and, also, to place minimum selling prices on the two lotteries (with an incentive-compatible revelation mechanism). A preference reversal occurs when a subject chooses one lottery in a pair but places the higher selling price on the other lottery. With experimental designs that discriminate between intransitivities and response mode (choice or valuation) effects, the data show that most preference reversals cannot be attributed to

\footnotetext{
${ }^{3}$ Principles for design of theory-testing experiments must be based on theory regardless of whether we do or do not expect the data to be consistent with theory. Some, at least informal, theory is needed to design heuristic experiments intended to produce stylized facts; in the absence of any theory we don't even know what variables to record as "data" in an experiment.
} 
intransitivities (Tversky, Slovic, and Kahneman, 1990; Cox and Grether, 1996). Robust preference reversals that are a response mode effect would constitute a serious problem for economics because they would support the conclusion that choice tasks elicit different preferences than do valuation tasks. ${ }^{4}$ But suppose that an experimenter were to pay subjects for both tasks at the end of the experiment. In that case, an observed "preference reversal" of the above type would not be a problem for standard theory because it could result from a "portfolio effect” in the experiment. For example, suppose a risk averse subject prefers lottery $A$ to lottery $B$ and, given that returns in lotteries A and B are uncorrelated (because of experiment procedures), prefers the diversified portfolio $(A, B)$ to the undiversified portfolios $(A, A)$ and $(B, B)$. In that case, the apparent preference reversal could, instead, be an attempt to construct the individual's optimal diversified portfolio in the only way possible within the experiment: choose one lottery and place the higher selling price on the other lottery (because of preferring to keep this one to complete the portfolio).

Such possible portfolio effects can be avoided by realizing stochastic payoffs and crediting a subject's earnings after each decision. But this procedure changes a subject's wealth between decisions and introduces the possibility that an apparent preference reversal could be attributed to a change in risk preferences caused by the change in wealth, which is consistent with expected utility theory of choice under risk. It is not a problem for such theory if lottery $A$ is preferred to lottery $B$ at wealth $w_{1}$ while lottery $B$ is preferred to lottery $A$ at wealth $w_{2} \neq w_{1}$. In order to avoid both possible portfolio effects and wealth effects, an experimenter can follow the accepted practice of randomly selecting one of a subject's decisions for payoff. But the validity of this procedure for solving the possible problems in interpreting data depends on the independence

\footnotetext{
${ }^{4}$ As an example, consider the implausibility of conducting a cost-benefit analysis when: (a) project $A$ is better than project $B$ with choice task data; but (b) project $B$ is better than project $A$ with valuation task data.
} 
axiom of expected utility theory (Holt, 1986). ${ }^{5}$ It is the independence axiom that accounts for linearity in probabilities of the expected utility functional, which implies that random selection of one decision for payoff will preserve the preference for $A$ over $B$ in all decision tasks.

If, for example, the dual independence axiom (Yaari, 1987) is assumed, rather than the independence axiom, then the utility functional is always linear in payoffs but is linear in (decumulative) probabilities only if the agent is risk neutral. If the dual axiom is assumed then possible wealth effects pose no problem for interpreting data, because of linearity in payoffs, but random selection of one decision for payoff can be a problem. With probability transformation, the relevant distribution is the transformed cumulative (or decumulative) distribution of payoffs from all of the decision tasks in the experiment. With a functional of this type, introducing random selection of one decision for payoff changes the utilities of multiple decision tasks in a fundamental way. This implication holds for the dual theory of expected utility (Yaari, 1989), rank dependent expected utility theory (Quiggin, 1993), and cumulative prospect theory (Tversky and Kahneman, 1992). ${ }^{6}$

\subsection{Current Relevance of Random Selection of a Decision}

The above discussion used experiments on the preference reversal phenomenon as an example to explicate some conventions of experimental design that have general implications. Some recent experiments reported in the literature reveal how these conventions, and their theoretical foundations, have current relevance. The recent papers discussed in following paragraphs are selected because they report experiments on important topics and incorporate

\footnotetext{
${ }^{5}$ In order to avoid confounding preference reversals with empirical inconsistencies with the independence axiom, Cox and Epstein (1989) and Cox and Grether (1996) credited subjects' earnings after each decision and, subsequently, used econometric analysis of data to ascertain whether apparent preference reversals could be attributed to wealth effects. There were no significant wealth effects; hence the data provide tests for preference reversals that are not confounded with violations of either the independence axiom or the dual axiom.

${ }^{6}$ Tversky and Kahneman (2000, pp. 25-26) maintain that, as an empirical result, in some contexts subjects isolate on individual decision tasks in multiple task experiments. Such behavior is inconsistent with rank dependent theories, including cumulative prospect theory.
} 
experimental designs commonly used in the literature, not because these papers should be singled out for special criticism.

Recent papers report experiments that introduce role reversal into the investment game (Burks, Carpenter, and Verhoogen 2003; Chaudhuri and Gangadharan, 2007) in order to test for effects of a design change intended to promote subjects' understanding of the game. Subjects are paid for both (first mover and second mover) decisions at the end of the experiment, hence there is a possible portfolio effect that confounds comparison of the role-reversal data to data from single decision experiments with the investment game without role reversal. As a consequence, the effects of role reversal, per se, cannot be indentified with data from the experiment. An alternative design would use random selection of one of the two decisions in the role reversal treatment for payoff. Given the independence axiom, this would solve the possible portfolio effects problem. Without the independence axiom, however, or with an alternative such as the dual independence axiom, random selection of one decision for payoff would not solve all confound problems, for reasons explained above.

Another example is provided by recent experiments on risk attitude. Holt and Laury (2002, 2005), Harrison, et al. (2005), and Eckel and Grossman (2002, 2008) ask subjects to make several choices between alternative lotteries in order to measure their risk attitudes. One decision is randomly selected for payoff in order to avoid confounds from possible portfolio or wealth effects. As explained above, the theoretical foundation for this method is the independence axiom of expected utility theory; interpretation of data from these experiments is different if the subjects are not expected utility maximizers. If, for example, we assume the dual independence axiom rather than the independence axiom then the probability distribution in the utility functional is the transformed cumulative (or decumulative) distribution of payoffs from all of the decision tasks in the experiment, compounded with the distribution for random decision selection. In that case, for example, the row in the Holt-Laury decision task table at which a subject switches from one lottery to another has a different interpretation for a subject's revealed risk attitude. Given the 
dual axiom, one should pay off after every decision, since wealth effects are irrelevant, and thereby avoid aggregating risks associated with the decision selection lottery with all of the underlying lotteries included in the decision tasks. This alternative of paying after every decision is also appropriate for other decision theories, such as prospect theory, in which the utility functional is defined on income rather than terminal wealth.

\subsection{Relevance to the Social Context Experiment}

The experiment in Cox (2000), cited by Smith (2008, p. 23), used random selection of one of two decision tasks for payoff and a double blind payoff protocol. Introduction of the second task had no significant effect on first movers' behavior in the investment game but significantly shifted behavior of second movers in the investment game and dictators in both the first-mover dictator-control game and the second-mover dictator-control game towards more generosity. One possible explanation of this finding is that subjects' behavior is inconsistent with the independence axiom and that this accounts for the effect of existence of the second task on first task choices. This possible explanation could, of course, be checked by repeating the experiment with one change: inform the subjects that they will be paid between the first task and the second, as-yet-undefined decision task in the two-task treatment. If the difference between the one-task and two-task treatments were to disappear with this design then one could attribute the social context effect to inconsistency of behavior with the independence axiom (and consistency with the dual axiom). If the difference between the one-task and two-task treatments were to be robust to this change in payoff procedures then we would have to look for other possible explanations that create interdependence between sequential decision opportunities.

An interesting candidate explanation is that existence of the second task induces a generalized "trusting" response in the first task, in which subjects try to encourage more generous behavior by others in the second decision task. This explanation can accommodate the insignificant change in first mover behavior in the investment game by postulating that the 
generalized trusting behavior does not significantly reinforce the specific first-mover trusting behavior observed for first movers in the single-task investment game.

\section{Context and Separability}

Many experiments on choice under certainty (in a "commodity space”) involve tests or applications of utility theories. Analysis of data from such experiments typically depends on an (usually implicit) assumption of separability: (a) within parts of an experiment; and (b) between the experimental environment and the outside world. First consider the question of withinexperiment separability or nonseparability.

\subsection{Reciprocal Preferences}

Many fairness games studied in experiments have an extensive form in which a first mover takes some action with implications that are observed by the second mover before the second mover takes action. There are many widely-studied games with this form. ${ }^{7}$ In some such games, second movers' decisions are responsive to first movers' decisions in ways known to support existence of reciprocity (Cox, 2004; Cox and Deck, 2005; Cox, Sadiraj, and Sadiraj, 2008; Falk, Fehr, and Fischbacher, 2008). Such reciprocal behavior is inconsistent with Neoclassical preference theory (Hicks, 1937; Samuelson, 1946) in which preferences are a characteristic of the agent that is independent of the environment and of actions by other agents. Reciprocal behavior is also inconsistent with models of unconditional social preferences such as Fehr and Schmidt (1999), Bolton and Ockenfels (2000), and (the text model in) Charness and Rabin (2002) in which the other-regarding preferences are a fixed characteristic of the agent. This is a manifestation of the "independence of history and future" property of theory discussed by Smith (2008).

\footnotetext{
${ }^{7}$ Games with this form include the ultimatum game, mini-ultimatum game, investment game, moonlighting game, Stackelberg duopoly game, carrot and/or stick games, power to take game, gift exchange game, and sequential VCM game.
} 
Recently, some progress has been made in incorporating "dependence on history” within an experiment into theory with development of models of reciprocity that are supported by data from many extensive form fairness games (Cox, Friedman, and Gjerstad, 2007; Cox, Friedman, and Sadiraj, 2008). In this theory, "reciprocity" depends both on prior actions by another person (Axiom R) and the context or circumstances in which those actions were chosen (Axiom S). Axiom S discriminates between acts of commission, acts of omission, and absence of opportunity to act in modeling reciprocity. This axiom also incorporates status quo ante a first mover's decision into the theory. In these ways, this theory incorporates "circumstances” of action (Smith, 2008, pp. 1-2) into modeling reciprocity. But “dependence on future” has still largely eluded theorists, while data provide support for such dependence in the form of experimental identification of trusting behavior by first movers in extensive form games (Cox, 2004; Cox and Deck, 2005; Cox, Sadiraj, and Sadiraj, 2008).

\subsection{Incomplete Data and Separability}

Although it is common to model nonreciprocal and reciprocal behavior within experiments with utility theory, it is not common to recall the independence assumptions that are (implicitly) being made, even though literature on the generalized axiom of revealed preference (GARP) is quite specific about this. Literature about GARP explores issues of separability of preferences and

completeness of data that have general relevance to experimental methods for choice on a commodity space.

Afriat (1967) and Varian (1982) demonstrate that GARP is the necessary and sufficient condition for the existence of a utility function that rationalizes observations of quantity and price vectors. But Varian (1988) explains that the utility maximization hypothesis has no testable implication for observations of a proper subset of the commodities chosen by a consumer. Furthermore, Varian (1983) explains that utility maximization, when a proper subset of 
commodities is weakly separable in an individual's preference ordering, is equivalent to GARP inequalities holding for both the weakly separable subset and for all of the commodities.

Accordingly, when we ask whether a subject's behavior in a typical laboratory or field experiment can be rationalized by a utility function, either in the absence of opportunities for reciprocity (Andreoni and Miller, 2002; Harbaugh, Krause, and Berry, 2001; Sippel, 1997; Mattei, 2000; Février and Visser, 2004) or in their presence (Cox, Friedman, and Gjerstad, 2007; Cox, Friedman, and Sadiraj, 2008), we are assuming (at least weak) separability between choices made within and outside the experiment. ${ }^{8}$ In the absence of such separability, the utility hypothesis has no testable implication for data from a typical laboratory or field experiment because the experiment produces observations of a proper subset of the economic choice variables that can affect utility (Cox, 1997).

An implication of the analysis in Varian (1983) is that we can use data from (non-closed economy) laboratory and field experiments to test only one of the necessary conditions for utilitymaximizing behavior but we cannot test sufficient conditions because the data needed do not exist. The necessary condition we can test for non-reciprocal preference theories is that GARP inequalities are satisfied for choice variables observed in the experiment. ${ }^{9}$ The necessary condition we cannot test is that GARP inequalities hold for all choice variables that enter budget constraints within and outside the experiment. Sufficient conditions for the compound hypothesis of weak separability and utility maximization are that GARP inequalities are satisfied for both the choice variables in the experiment (the separable subset) and all choice variables (within and outside the experiment). With data from almost all experiments, only one of the necessary

\footnotetext{
${ }^{8}$ There is a more serious problem in Sippel (1997). The experiment reported in that paper had two linear constraints, the usual budget constraint and a second constraint on time allocated to consumption activities. With two linear constraints, that sometimes intersect and sometimes do not intersect, GARP is not known to be necessary or sufficient for utility-maximizing behavior. Hence tests of such data for GARP violations have no known implication.

${ }^{9}$ With or without separability, standard GARP inequalities cannot be applied to test reciprocal preference theory because of its distinct implication that opportunities not chosen place restrictions on preferences (Cox, Friedman, and Sadiraj, 2008, pgs. 42-45).
} 
conditions can be tested. ${ }^{10}$ Finding low rates of violation of the one type of necessary condition is an important result, but it does not support the conclusion that the subject's behavior can be rationalized by a utility function. This is a prominent example that illustrates Smith's (2008, p.2) point that we are often not careful about distinguishing between "if-then" and "if-and-only-if statements.”

10 One known exception are data from the closed economy experiment reported in Battallio, et al. (1973), as analyzed in Cox (1997). But such data are extremely hard to generate and may contain significant subject selection effects because experimental closed economies are typically populated by incarcerated individuals such as residents of mental hospitals or prisons. 


\section{References}

Afriat, Sidney, “The Construction of Utility Functions from Expenditure Data,” International Economic Review, 8, 1967, 67-77.

Andreoni, James and John Miller, “Giving According to GARP: An Experimental Test of the Consistency of Preferences for Altruism,” Econometrica, 70(2), 2002, 737-753.

Battallio, Raymond C., John. H. Kagel, Robin C. Winkler, Edwin B. Fisher, Robert L. Basmann, Jr., and Leonard Krasner, “A Test of Consumer Demand Theory Using Observations of Individual Consumer Purchases,” Western Economic Journal, 11, 1973, 411-428.

Berg, Joyce, John Dickhaut, and Kevin McCabe, “Trust, Reciprocity, and Social History,” Games and Economic Behavior, 10(1), 1995, 122-142.

Bolton, Gary E. and Axel Ockenfels, "ERC: A Theory of Equity, Reciprocity, and Competition,” American Economic Review, 90, 2000, 166-193.

Burks, Stephen V., Jeffrey P. Carpenter, and Eric Verhoogen, "Playing Both Roles in the Trust Game,” Journal of Economic Behavior and Organization, 51(2), 2003, 195-216.

Chaudhuri, Ananish and Lata Gangadharan, “An Experimental Analysis of Trust and Trustworthiness,” Southern Economic Journal, 73(4), 2007, 959-985.

Charness, Gary and Matthew Rabin, “Understanding Social Preferences with Simple Tests,” Quarterly Journal of Economics, 117, 2002, 817-869.

Cox, James C., “On Testing the Utility Hypothesis,” Economic Journal, 107, 1997, 1054-1078.

Cox, James C., “Trust and Reciprocity: Implications of Game Triads and Social Contexts,” Working Paper 00-11, Department of Economics, University of Arizona, 2000; revised version forthcoming in New Zealand Economic Papers: Special Issue on Experimental Economics.

. Cox, James C., "How to Identify Trust and Reciprocity,” Games and Economic Behavior, 46(2), 2004, 260-281. 
Cox, James C. and Cary A. Deck, “On the Nature of Reciprocal Motives,” Economic Inquiry, 43(3), 2005, 623-635.

Cox, James C. and Seth Epstein, "Preference Reversals Without the Independence Axiom,” American Economic Review, 79(3), 1989, 408-426.

Cox, James C., Daniel Friedman, and Steven Gjerstad, “A Tractable Model of Reciprocity and Fairness,” Games and Economic Behavior, 59, 2007, 17-45.

Cox, James C., Daniel Friedman, and Vjollca Sadiraj, “Revealed Altruism,” Econometrica, 76(1), 2008, 31-69.

Cox, James C. and David M. Grether, “The Preference Reversal Phenomenon: Response Mode, Markets, and Incentives,” Economic Theory, 7(3), 1996, 381-405.

Cox, James C., Klarita Sadiraj, and Vjollca Sadiraj, “Implications of Trust, Fear, and Reciprocity for Modeling Economic Behavior,” Experimental Economics, 11, 2008, 1-24.

Eckel, Catherine C. and Phillip J. Grossman, “Sex Differences and Statistical Stereotyping in Attitudes toward Financial Risk,” Evolution and Human Behavior, 23 (4), 2002, 281 295.

Eckel, Catherine C. and Phillip J. Grossman, “Forecasting Risk Attitudes: An Experimental Study of Actual and Forecast Risk Attitudes of Women and Men,” Journal of Economic Behavior and Organization, 2008 (forthcoming).

Falk, Armin, Ernst Fehr, and Urs Fischbacher, “Testing Theories of Fairness -Intentions Matter,” Games and Economic Behavior, 62, 2008, 287-303.

Fehr, Ernst and Klaus M. Schmidt, “A Theory of Fairness, Competition, and Cooperation,” Quarterly Journal of Economics, 114, 1999, 817-868.

Février, Philippe and Michael Visser, “A Study of Consumer Behavior Using Laboratory Data,” Experimental Economics, 7(1), 2004, 93-114.

Grether, David M. and Charles R. Plott, “Economic Theory of Choice and the Preference Reversal Phenomenon,” American Economic Review, 69, 1979, 623-638. 
Harbaugh, William.T., Kate Krause and Timothy R. Berry, “GARP for Kids: On the Development of Rational Choice Behavior,” American Economic Review, 91, 2001, 1539-1545.

Harrison, Glenn W., Eric Johnson, Melayne McInnes, and Elisabet Rutström, "Risk Aversion and Incentive Effects: Comment,” American Economic Review, 95 (3), 2005, 897-901.

Hicks, John, Value and Capital. Oxford: Clarendon Press, 1939.

Holt, Charles A., "Preference Reversals and the Independence Axiom,” American Economic Review, 76, 1986, 508-514.

Holt, Charles A. and Susan K. Laury, “Risk Aversion and Incentive Effects,” American Economic Review, 92, 2002, 1644 - 1655.

Holt, Charles A. and Susan K. Laury, "Risk Aversion and Incentive Effects: New Data without Order Effects,” American Economic Review, 95, 2005, 902 - 912.

Mattei, Aurelio, “Full-scale Real Tests of Consumer Behavior using Experimental Data,” Journal of Economic Behavior and Organization, 43(4), 2000, 487-497.

Quiggin, John, Generalized Expected Utility Theory: The Rank-Dependent Model. Boston: Kluwer Academic Publishers, 1993.

Samuelson, Paul A. Foundations of Economic Analysis. Cambridge, MA: Harvard University Press, 1947.

Sippel, Reinhard, “An Experiment on the Pure Theory of Consumer’s Behaviour,” Economic Journal, 107, 1997, 1431-1444.

Smith, Vernon L., “Theory and Experiment: What are the Questions?”, Journal of Economic Behavior and Organization, 2008 (forthcoming).

Tversky, Amos and Daniel Kahneman (eds.), Choices, Values, and Frames, Cambridge University Press, 2000.

Tversky, Amos, Paul Slovic, and Daniel Kahneman, “The Causes of Preference Reversal,” American Economic Review, 80, 1990, 204-217. 
Varian, Hal R., “The Nonparametric Approach to Demand Analysis,” Econometrica, 50, 1982, 945-972.

Varian, Hal R., “Non-parametric Tests of Consumer Behavior,” Review of Economic Studies, 50, 1983, 99-110.

Varian, Hal R., "Revealed Preference with a Subset of Goods,” Journal of Economic Theory, 46, $1988,179-189$.

Yaari, Menahem E., “The Dual Theory of Choice under Risk,” Econometrica, 55, 1987, 95-115. 